\title{
La religión como predictor de las actitudes hacia la nación
}

\author{
José Miguel Rodríguez García \\ Instituto de Investigaciones Psicológicas \\ Universidad de Costa Rica
}

Ce:jomiguelrg@gmail.com

\begin{abstract}
Resumen. En éste articulo se analizan las relaciones entre religiosidad y nacionalismo. Específicamente se analizan las correlaciones entre variables como identidad nacional, nacionalismo, patriotismo, fundamentalismo religioso, religiosidad, así como el efecto predictor de las variables religiosas sobre las actitudes políticas hacia la nación. Para ello se realizó una encuesta con participantes costarricenses. Los resultados del estudio se discuten tanto desde la perspectiva de las implicaciones para la investigación psicosocial como desde el punto de vista de la investigación del nacionalismo en Costa Rica.
\end{abstract}

Palabras clave: nacionalismo, religiosidad, identidad nacional, patriotismo, fundamentalismo religioso.

\begin{abstract}
In this article the relations between religiosity and nationalism are analyzed. Specifically the correlations between variables like national identity, nationalism, patriotism, religious fundamentalism, and religiosity; as well as the predicting effect of the religious variables are analized on political attitudes toward the nation. It was performed a survey with Costa Ricans participants. The results are discussed from the point of view of psychosocial research as from the point of view of the research on nationalism in Costa Rica.
\end{abstract}

Key Words: Nationalism, religiosity, national identity, patriotism, religious fundamentalism. 


\section{Introducción}

Uno de los temas que han cobrado mayor importancia en la discusión académica en los últimos años en las ciencias sociales costarricenses es lo que se podría llamar a grandes rasgos el problema de la identidad. Diversas publicaciones en disciplinas como la historia, los estudios culturales, la psicología, la literatura y la filosofía por señalar solamente las más evidentes, se han dado a la tarea de pensar el problema de la identidad o las identidades, colectivas o personales de los y las costarricenses. Tales estudios tratan de aportar información a la pregunta de cómo se configura la identidad en Costa Rica en los tiempos actuales. Esto es en parte resultado del acelerado proceso de globalización económica y los aspectos culturales relacionados con este proceso.

El presente artículo se ocupa de analizar desde le punto de vista de la psicología social cuál es la contribución de la religión a una de esas formas de identidad colectiva. Específicamente analiza la influencia de la religión en uno de los componentes de la construcción de la idea de nación en Costa Rica, el nacionalismo. Por lo anterior se revisan algunas de las discusiones presentes en las ciencias sociales contemporáneas y específicamente en la psicología social, para evaluar posteriormente la influencia de la religión sobre el nacionalismo en Costa Rica por medio de una investigación empírica.

\section{Nacionalismo y religión}

En la historia, las relaciones entre nacionalismo y religión son en algunos casos más claras que en otros. Ésta relación se escenifica de formas muy evidentes como es el caso del catolicismo en Irlanda y el judaísmo en Israel. Pero aún en estos casos es evidente que la religión no es el único motor de las identificaciones nacionales, si no que se debería entender como un componente más de un complejo tramado de relaciones donde acaso el papel de la religión sobre el nacionalismo es evidente pero de ninguna manera el único.

La nación es entendida aquí siguiendo a Gellner $(1989,1997,1998)$ y Baumann (2001) como una unidad política que es consecuencia del accionar de un Estado que aspira a convertirse en un Estado-nación. En otras palabras, una nación es una construcción ideológica que es estimulada por una organización política que pretende establecer una coherencia entre cultura y Estado. Para ello, los estados recurren al nacionalismo, que es un principio político que supone la transformación de los valores y la creación de nuevos referentes. Este proceso de transformación es mediatizado por las 
instituciones sociales que facilitan la concreción de un discurso político homogenizador en una población naturalmente heterogénea.

De tal manera el nacionalismo es un mecanismo que sustenta la idea de nación con la exaltación de elementos tales como la lengua, la etnicidad, la religión, y la conciencia de pertenecer a una entidad política duradera. Se trata de criterios que permiten distinguir entre "nosotros" y "ellos", y que por lo tanto imprimen una forma de identificación para el grupo interno y el externo. En otras palabras define quién es miembro de esa comunidad imaginada (Anderson, 1991) que se llama nación y cuáles son las calidades de sus miembros.

En síntesis la nación como construcción ideológica es un producto de estrategias nacionalistas, las cuales a su vez son producto del accionar de organizaciones políticas que pretenden crear una unidad entre la cultura o las culturas de una población y tal organización política. En pocas palabras, la construcción de una nación es el resultado de un Estado-nación.

Por otra parte, algunas de estas características no son exclusivas del sistema político y pueden ser homologables en otros sistemas sociales como es el caso de la religión, con la diferencia de que su discurso no está centrado en la consolidación de un Estado Nacional, ubicado físicamente en un territorio, pero sí en la homogenización de grandes masas de población ante una misma idea de la trascendencia.

Por su parte religión es entendida aquí desde una acepción ubicada en las ciencias sociales, dejando de lado otras posibles implicaciones, especialmente de carácter teológico. Esto implica usar una definición de trabajo. En esta investigación, religión es entendida como un sistema de creencias y prácticas por las cuales un grupo de personas interpreta y responde a lo que ellos sienten que es sagrado y sobrenatural Johnstone, 1991). En este sentido la religión aunque tiene un correlato individual es un fenómeno grupal y por lo tanto es una forma de interacción y de comunicaciones entre grupos de personas, hay metas y normas compartidas, hay roles, funciona colectivamente mediante un sistema de status, y hay un sentido de identificación con el grupo. Sin embargo, no se trata de un grupo cualquiera sino que está concentrado en lo sobrenatural o lo sagrado, sobre lo cual desarrolla un cuerpo de creencias, un conjunto de prácticas e involucra una serie de prescripciones morales.

Como señala Armstrong (1997) tanto religión como nacionalismo constituyen dos discursos que conectan a diferentes generaciones y diferentes grupos de personas. Tanto la religión como el nacionalismo constituyen sistemas de pensamiento que homogenizan a las masas, postulando una herencia y una historia compartidas, así como un destino común. Tales características hacen que la nación y la religión constituyan recursos que permiten resguardar y conformar la identidad personal y la colectiva. Para este autor estos elementos son particularmente evidentes en 
los periodos de grandes transformaciones sociales donde con frecuencia está bajo amenaza la identidad. En tales situaciones tanto religión como nación se manifiestan como muros de contención que sostienen la identidad frente a la amenaza de la anomia.

En el nivel del funcionamiento también se pueden encontrar similitudes. Tanto el Estado nacional como la organización religiosa son modelos de autoridad, órdenes de poder que promueven una forma de relación en las personas o modelos de desarrollo de la vida cotidiana indicando las posibilidades y los límites de la actividad social. Además, ambas formas de estructuración parten de un principio de homogeneidad, por lo que todos los miembros de una comunidad religiosa son iguales entre sí de la misma manera que los miembros de una nación son iguales entre sí en tanto que miembros de tal comunidad. Adicionalmente nacionalismo y religión como formas de identificación recurren a elementos simbólicos como mediadores de las relaciones entre los individuos y las creencias estimuladas por las organizaciones. Las fiestas patrias así como las fiestas y actividades religiosas son prácticas ritualizadas en las que se renuevan y reproducen las creencias, los valores y las normas, tanto de la religión como de la nación.

A pesar de las similitudes anteriores es importante señalar que no se puede igualar una identificación religiosa con una identificación nacional. Las investigaciones historiográficas explican que si bien la religión es un factor que está relacionado con la identidad nacional, la forma en la que se relacionan así como la importancia de tal relación es relativa a los diferentes contextos sociales o a la historia de los estados. Es decir, la forma en la que se vinculan religión y nación es un resultado de particularidades sociales y momentos históricos, un resultado del encuentro entre espacio, tiempo y sociedad.

Esta última observación está especialmente presente en el trabajo de Hobsbawn (1991), donde ubica a la religión como un componente protonacionalista que comprende sentimientos nacionalistas en el ámbito popular sobre los cuales se puede configurar un nacionalismo político. Esto implica que si bien la religión es un componente sobre el cual los estados pueden articular un discurso ideológico, no es éste el único y dependiendo del contexto tampoco el más relevante. Otros componentes protonacionales como la lengua, la etnicidad o la conciencia de pertenecer o haber pertenecido a una entidad política son también explotados e incorporados como recursos aprovechables en la concreción de una nación.

Es importante señalar que las relaciones entre nación y religión también pueden ser observadas de acuerdo a las diferentes intensidades con las que estas se manifiestan. Es posible pensar en continuos donde la relación entre nación y religión es muy espuria hasta contextos donde ambos elementos están altamente imbricados. Un caso extremo lo constituiría lo que Friedland (2001) ha llamado nacionalismo religioso, donde el nacionalismo estaría 
definido a través de la religión. En esta manifestación la política se constituye en una obligación religiosa y la religión es la base para el razonamiento político y la construcción de la identidad. Este sería el caso de estados confesionales en donde la organización religiosa y la organización política no se diferencian. Del mismo modo cabe suponer que hay grados en un continuo que anteceden al nacionalismo religioso hasta llegar a un estado secular donde la religión si bien forma parte de la sociedad es un elemento completamente diferenciado y subordinado al Estado. Una aproximación a esta continuidad es presentada por Johnstone (1992). Para este autor en un extremo estaría la teocracia pura donde el Estado es guiado directamente por Dios por medio de sus profetas o elegidos. En el extremo opuesto estaría el sistema totalitario, donde la religión es controlada por el Estado y la usa como una herramienta más. En medio existirían diferentes posibilidades como la teocracia modificada, la separación parcial, y la total separación entre Estado y religión.

En el contexto costarricense la relación entre nacionalismo y religión no ha sido la más privilegiada por la investigación social. Si bien existen algunos elementos dispersos en investigaciones historiográficas, los análisis sistemáticos son escasos. Una de las pocas excepciones a lo anterior es el trabajo de González (1994). En uno entre profano y sagrado no resulta tan clara a pesar de la secularización del mundo que se construye el discurso histórico de la nación costarricense. Tal análisis plantea como resultado que la legitimación del Estado costarricense ha dependido en parte del uso de elementos religiosos. Sin embargo, la tesis más fuerte en esta relación es que, de acuerdo a los resultados de González (1994), en la superposición de los discursos políticos y religiosos se encuentra el origen histórico de la identidad nacional costarricense. De esta manera se podría plantear que el nacionalismo, entendido como un discurso político se entremezcla con el discurso religioso dando como resultado un mecanismo de legitimación del Estado que lo trasciende, intentando sacralizarlo. Así pues existen razones para pensar en una relación entre las identificaciones nacionales y la religión. De ser acertadas estas proposiciones sería esperable entonces encontrar correlatos de la misma al nivel grupal e individual.

\section{Nacionalismo y religión desde la psicología social}

Tanto nacionalismo como religión han sido objeto de atención por la investigación psicosocial desde 1950, principalmente en Estados Unidos; ambos en su conexión con el prejuicio étnico o con la personalidad autoritaria (Adorno, 1950; Allport, 1954). En el caso del nacionalismo, tal relación se estudió principalmente desde el punto de vista del etnocentrismo y en el caso de la religión el foco del análisis se puso en la intensidad de la vinculación religiosa como predictor de diversos tipos de prejuicio. Síntesis 
narrativas de la relación entre prejuicio y religión pueden observarse en Batson, Schoenrade y Ventis (1993) y en Hunsberger y Jackson (2005).

En un influyente capítulo Kelman (1997), siguiendo las distinciones de Feschbach (1994) define la identidad nacional como un vínculo emocional y subjetivo a la nación. La identidad nacional es una definición de un grupo en tanto grupo. Esta definición comprende características y valores, fortalezas y debilidades, reputación y condiciones de existencia, instituciones y tradiciones, historia y objetivos. La identidad nacional está presente en los individuos pero es diferente a la suma de las concepciones de los individuos. Por un lado porque tiene una existencia independiente en diferentes formas de registro (documentos históricos, diferentes producciones culturales, etc.), pero también por los diferentes grados en que se involucran y participan sus miembros. Evidentemente se trata de un fenómeno social que es reproducido por medio de la socialización en sus diferentes medios y espacios.

La identidad nacional ha sido estudiada recientemente por la psicología social tomando en cuenta sus principales componentes que son el nacionalismo y el patriotismo. Ambos implican formas de vinculación a la nación o de tener una identidad nacional pero difieren en cómo ésta vinculación se lleva a cabo. Estas diferencias se pueden entender desde la teoría de la identidad social. Siguiendo la interpretación de Mummendy, Klink y Brown (2001) patriotismo se entiende como una manera de evaluación intragrupal mientras que nacionalismo como una manera de diferenciación grupal. Es decir que el patriotismo consiste en una vinculación autónoma donde los miembros del grupo evalúan a la nación, mientras que el nacionalismo es una evaluación que se realiza con referencia a grupos externos y por medio de esta evaluación se construye una afirmación de la identidad.

Entonces, el nacionalismo es una idealización de la propia nación que implica una evaluación positiva de ésta, un sentimiento de superioridad nacional y una aceptación acrítica del Estado nacional y de las autoridades nacionales. Presenta un gran énfasis en los auto-conceptos individuales y en la reducción de las actitudes ambivalentes hacia la nación. Hay una inclinación a evaluar a los miembros de la nación en función de la descendencia, la raza, o el grupo cultural de referencia y a considerar a la nación como un grupo homogéneo. Adicionalmente todos estos elementos son decisivos en las evaluaciones que se hace de otros grupos nacionales.

El patriotismo, en cambio, es entendido como una vinculación menos ingenua a la nación. La nación no es idealizada sino que es evaluada con conciencia crítica o de una manera independiente de la opinión de las élites del estado o de los discursos establecidos. Esto implica que el apoyo a los fines del sistema, así como a los objetivos de la nación no va más allá que los límites de los valores humanos. Patriotismo como dimensión incluiría el 
apoyo a los principios democráticos y el rechazo a las formas autoritarias de gobierno. La historia nacional es examinada desde diferentes puntos de vista y considerando las perspectivas de los diferentes grupos participes de ella.

Evidentemente estas conceptuaciones implican una polarización de dos posibilidades de aproximarse a la nación, sin embargo, otras consideraciones son necesarias. Por un lado ambas posibilidades no son excluyentes por lo que personas que sostienen actitudes nacionalistas también pueden presentar actitudes patriotas en algún grado, por lo que más que un continuo constituyen aproximaciones paralelas o diferentes dimensiones de un mismo fenómeno. Esta distinción teórica ha sido trasladada a la investigación empírica teniendo como resultado la diferenciación de estas dos dimensiones (Schatz, Staub \& Lavine, 1999; Feschbach, 1994; Blank \& Shmidt, 2003; Schatz \& Staub, 1997; Hjerm, 1998; De De Figueredo \& Elkins, 2003; Mummendy, Klink \& Brown; 2001) aunque en las diferentes investigaciones realizadas es una distinción que no mantiene una nomenclatura homogénea (una discusión detallada sobre los alcances y diferencias conceptuales de estas dimensiones se puede encontrar en Cohrs, 2005).

\section{Resultados de investigaciones empiricas.}

Por su parte en la investigación en psicología social pocos estudios empíricos se han enfocado en analizar las relaciones entre nacionalismo y religión, aunque se encuentran resultados empíricos al respecto; entre estas investigaciones se pueden señalar las siguientes: Levinson, (1957) Martin y Westie, (1959) encuentran correlaciones entre nacionalismo y medidas de religiosidad. En ambos casos tal relación es explicada señalando que esta relación es esperable en tanto la religiosidad como el nacionalismo son entendidos como manifestaciones de conservadurismo. Eisinga, Felling y Peters (1990a y 1990b) discuten esta relación en el contexto de los Países Bajos. De acuerdo con sus resultados las creencias religiosas tienen un efecto positivo en el nacionalismo. Esta relación se confirmó en las diferentes formas de religiosidad que fueron medidas en el estudio con excepción de la parte de la muestra que mostró mayor intensidad religiosa y aquella parte de la muestra que evidenció no tener ningún vínculo religioso. En ambos casos no se encontró relación alguna. En otras palabras, únicamente los participantes con una vinculación religiosa intermedia entre los no creyentes y los muy creyentes mostraron correlaciones positivas y significativas entre religiosidad y nacionalismo.

Schatz \& Staub (1997) analizan la relación entre Búsqueda Religiosa y Religiosidad Intrínseca con las dimensiones de Patriotismo Ciego y 
Patriotismo Constructivo ${ }^{1}$. Ambas formas de vinculación nacional están positivamente correlacionadas con la orientación intrínseca a la religión, pero Patriotismo Ciego esta correlacionado negativamente con la escala religiosa de Búsqueda ${ }^{2}$. Haciendo eco de los planteamientos de Rockeach (1960) estos resultados podrían sugerir que las formas de pensamiento crítico hacia la religión se contraponen a las formas de pensamiento más rígido que estarían presentes en el nacionalismo. Sin embargo no se encontraron correlaciones entre la escala de Búsqueda y Patriotismo Constructivo, como era esperable teóricamente.

Más recientemente Burris, Branscome y Jackson (2000) encontraron que la orientación intrínseca a la religión estaba relacionada con componentes ideológicos de los estereotipos nacionales. Esto significa que la actitud hacia la nación está relacionada con las creencias religiosas de las personas, particularmente con aquellas que ven en la religión un fin en sí mismo, en la construcción del discurso nacionalista por parte de los estados, el fervor religioso es aprovechado por medio de la sacralización de la nación y los símbolos nacionales. Burris et al. encontraron que la orientación religiosa estaba relacionada con aquellos aspectos explícitamente ideológicos de los auto-estereotipos nacionales.

Duriez, Luyten, Snauwaert y Hutsebaut (2002) han analizado la relación entre nacionalismo y religión aplicando la Escala de Creencia Post-Crítica. En este estudio encuentran que ninguna de las cuatro dimensiones de la religiosidad por ellos estudiadas (ortodoxia, crítica externa, relativismo y segunda ingenuidad) correlaciona con el nacionalismo. Sin embargo, en este mismo estudio la medida de asistencia a la iglesia está positiva y muy significativamente correlacionada con nacionalismo, y en un modelo de regresión se constituye en un predictor de éste.

Finalmente y con una posición más integral, Altemeyer (2003) construyó una medida que sintetiza la relación entre nacionalismo y religión en la dimensión de etnocentrismo religioso. Esta investigación ubica al pensamiento religioso como parte de los elementos con los cuales se identifican y diferencian los grupos. Esta medida correlaciona con fundamentalismo religioso y con otras medidas como prejuicio y etnocentrismo.

En síntesis, la relación entre identificaciones nacionales y religión no han sido las privilegiadas en la investigación psicosocial. Como se puede ver no hay mucha investigación empírica sobre esta relación a pesar de que tanto

\footnotetext{
${ }^{1}$ En la terminología de Schatz y Staub (1997), Patriotismo ciego es un equivalente de nacionalismo y Patriotismo Constructivo de patriotismo, como han sido expuestos en este texto, aquí sin embargo se respeta la nomenclatura original.

2 Una amplia discusión de diversas escalas psicológicas para medir religión se encuentra en Batson, Schoenrade y Ventis (1993). Particularmente se discuten entre otras la relevancia de la escala de búsqueda y la orientación intrínseca y extrínseca hacia la religión.
} 
nacionalismo como religión han sido estudiados en relación con otra gran variedad de aspectos. Posiblemente se deba a que esta no es una relación que parezca ser tan obvia como religión y conservadurismo o nacionalismo y prejuicio. Ahora bien, precisamente por ello es que una investigación de tal relación parece necesaria.

Con los elementos expuestos hasta aquí podemos proponer cuatro hipótesis en la que se centra la presente investigación. Dado que las formas de identificación con la nación y la religión teóricamente pueden estar vinculadas, y específicamente en el contexto costarricense resultados de investigación han sugerido tal relación, se espera que (hipótesis 1) el nacionalismo va a estar correlacionado con dos medidas de la dimensión religiosidad. De igual manera, (hipótesis 2) se espera que la identidad nacional va a estar correlacionada con religiosidad. Adicionalmente, se espera que a mayor religiosidad se presente mayor nacionalismo (hipótesis 3) y mayor identidad nacional (hipótesis 4), por lo que la religiosidad tendrá un efecto predictor sobre estas dos variables.

\section{Método}

\section{Participantes}

Las personas participantes para este estudio fueron obtenidas durante una encuesta aplicada a estudiantes de colegio y a sus progenitores en el mes de diciembre de 2004 en dos colegios del área urbana de San José, Costa Rica. Esta encuesta se aplicó con el fin de probar diversos instrumentos psicológicos que forman parte de un estudio más amplio sobre aculturación y prejuicio hacia inmigrantes y minorías étnicas en Costa Rica.

Del total de 179 cuestionarios recolectados se desecharon 15 debido a que los(as) entrevistados(as) eran minorías étnicas. Solamente se mantuvieron aquellos(as) entrevistados(as) que se identificaron a sî mismos(as) como costarricenses por nacimiento y como blancos(as) o mestizos(as). Contar solamente con personas que son costarricenses por nacimiento responde a que la investigación en nacionalismo sugiere que no observar esta condición en la selección de la muestra tienen efectos en los resultados (Hjerm, 1998). En total se contó entonces con 164 casos.

Los y las participantes conforman una muestra por conveniencia. Esta modalidad es utilizada con frecuencia en la investigación en psicología social y en psicología de la personalidad, principalmente porque el foco de interés es mostrar las relaciones estructurales entre las variables y no presentar resultados descriptivos para una población (Wegener \& Fabrigar, 2000). Con 
este tipo de muestreo se asume que tales relaciones pueden ser encontradas en otras muestras aleatorias.

El $62 \%$ de los respondientes fueron mujeres. El $56 \%$ de los entrevistados pertenecen a estratos socioeconómicos bajos. El 33\% de la muestra estuvo conformada por padres y madres de familia.

\section{Procedimiento}

Tanto progenitores como estudiantes fueron informados de los propósitos del estudio y se les solicitó su participación voluntaria en éste. La aplicación de los instrumentos estuvo realizada por dos asistentes de investigación adecuadamente instruidos para tal labor. El cuestionario a los y las estudiantes fue aplicado en el salón de clases y el de padres y madres fue llevado a la casa por medio de los y las estudiantes con una carta introductoria respecto al objetivo de la investigación y las instrucciones necesarias para completar la encuesta. En ese documento se adjuntó un teléfono y una dirección electrónica en caso de que los progenitores quisieran hacer alguna consulta o manifestar sus opiniones al respecto. La aplicación del instrumento duró en promedio 37 minutos $(\mathrm{SD}=5.78)$.

\section{Instrumentos}

Se reseñan a continuación las características de las escalas y medidas de interés para este estudio. Los detalles de los ítems, los datos descriptivos y psicométricos se pueden observar en la Tabla 1.

La identidad nacional, nacionalismo y patriotismo fue medida por medio de escalas desarrolladas por Blank y Schmidt (2003). Estas tres escalas fueron desarrolladas para el contexto alemán por lo que algunos ítems fueron eliminados, como por ejemplo "Debido a nuestra superioridad económica deberíamos dominar las decisiones internacionales" o ítems relacionados con el orgullo hacia la tenencia del ejército. Las tres escalas tienen opciones de respuesta tipo Likert con cuatro puntos de respuesta que van de 1 totalmente en desacuerdo / nada orgulloso / nada importante a 4 totalmente de acuerdo / muy orgulloso / muy importante.

La escala de identidad nacional está compuesta por tres ítems que miden la importancia de ser costarricense, poseer ciudadanía y tener un vínculo con Costa Rica. La escala de nacionalismo está conformada por seis ítems en los que se evalúa el grado de orgullo que se siente sobre diversos aspectos. Por su parte la escala de patriotismo está conformada por seis ítems en los que se evalúa el orgullo que se siente por las instituciones y aspectos autocríticos respecto al país. 
Tabla 1

Datos descriptivos y psicométricos para las escalas

\begin{tabular}{|c|c|c|c|c|c|}
\hline \multicolumn{2}{|c|}{$\begin{array}{l}\text { Escala de Identidad Nacional } \\
\text { (Blank \& Schmidt, (2003) }\end{array}$} & \multirow{2}{*}{$\begin{array}{l}\mathbf{M} \\
3,81\end{array}$} & \multirow{2}{*}{$\frac{\text { SD }}{, 53}$} & \multirow{2}{*}{$\frac{\mathbf{r}_{\mathrm{ti}}}{, 70}$} & \multirow{2}{*}{$\begin{array}{r}\text { Fac. } 1 \\
86\end{array}$} \\
\hline Ni01 & Para mí, ser ciudadano costarricenses es... & & & & \\
\hline NiO2 & Para mí tener un vínculo con Costa Rica significa... & 3,74 &, 57 &, 78 & ,91 \\
\hline $\mathrm{NiO3}$ & Para mí, poseer ciudadanía costarricense es... & 3,82 &, 51 & ,65 & ,83 \\
\hline & Escala completa & 11,36 & 1,35 & & \\
\hline \multicolumn{2}{|c|}{$\begin{array}{l}\text { Escala de Nacionalismo } \\
\text { (Blank \& Schmidt, 2003) }\end{array}$} & $\mathbf{M}$ & SD & $\mathbf{r}_{\mathrm{ti}}$ & Fac.1 \\
\hline Na01 & Cuanto diría usted que esta orgulloso de ser costarricense & 3,76 &, 56 &, 59 & ,74 \\
\hline $\mathrm{NaO2}$ & La historia costarricense me hace sentir... & 3,43 &, 74 & 60 & ,76 \\
\hline $\mathrm{Na03}$ & El éxito de Costa Rica en los deportes me hace sentir... & 3,47 &, 74 &, 58 &, 72 \\
\hline $\mathrm{Na} 04$ & $\begin{array}{l}\text { El hecho de que Costa Rica sea el numero uno en } \\
\text { Centroamérica me hace sentir... }\end{array}$ & 3,71 & ,63 & ,64 & ,77 \\
\hline $\mathrm{Na05}$ & Para mí, Costa Rica es el mejor país del mundo & 3,27 &, 87 & ,66 & ,79 \\
\hline \multirow[t]{2}{*}{$\mathrm{Na} 06$} & $\begin{array}{l}\text { La moral y los valores costarricenses deberían ser un } \\
\text { modelo a seguir por otras naciones }\end{array}$ & 3,00 & ,91 &, 47 & 60 \\
\hline & Escala completa & 20.64 & 3,20 & & \\
\hline \multicolumn{2}{|r|}{$\begin{array}{l}\text { Escala de Patriotismo } \\
\text { (Blank \& Schmidt, 2003) }\end{array}$} & $\mathbf{M}$ & SD & $\mathbf{r}_{\mathrm{ti}}$ & Fac.1 \\
\hline Pa01 & $\begin{array}{l}\text { Las instituciones democráticas costarricenses me hacen } \\
\text { sentir... }\end{array}$ & 2,72 &, 89 &, 53 & ,75 \\
\hline $\mathrm{Pa} 02$ & $\begin{array}{l}\text { El sistema de seguridad social de Costa Rica me hace } \\
\text { sentir... }\end{array}$ & 2,49 & ,94 &, 49 & ,74 \\
\hline $\mathrm{Pa} 03$ & $\begin{array}{l}\text { Las posibilidades de participación política en Costa Rica } \\
\text { me hacen sentir... }\end{array}$ & 2,49 & 1,03 &, 54 & ,76 \\
\hline $\mathrm{Pa} 04$ & $\begin{array}{l}\text { Cuando yo critico a Costa Rica lo hago a pesar de la lealtad } \\
\text { que siento por mi país }\end{array}$ & 3,11 & ,93 &, 36 &, 52 \\
\hline $\mathrm{Pa} 05$ & $\begin{array}{l}\text { Si uno siente lealtad por su país uno debería esforzarse por } \\
\text { arreglar los problemas }\end{array}$ & 3,43 & ,82 &, 34 &, 50 \\
\hline \multirow[t]{2}{*}{ Pa06 } & $\begin{array}{l}\text { Yo aprecio mucho el sistema democrático de Costa Rica } \\
\text { pero estoy dispuesto a criticarlo para que mejore }\end{array}$ & 2,72 & ,89 &, 31 & ,45 \\
\hline & Escala completa & 17.64 & 3.64 & & \\
\hline \multicolumn{2}{|c|}{$\begin{array}{l}\text { Escala de Afiliación Religiosa } \\
\text { (Linder \& Moore, 2002) }\end{array}$} & $\mathbf{M}$ & SD & $\mathbf{r}_{\mathrm{ti}}$ & Fac.1 \\
\hline $\mathrm{Re} 01$ & ¿Cuán importante es la religión para usted? & 3,35 & 1,00 &, 55 & 81 \\
\hline $\mathrm{ReO2}$ & $\begin{array}{l}\text { ¿Cuán frecuentemente asiste usted a los servicios o } \\
\text { actividades religiosas? }\end{array}$ & 2,80 & ,99 & ,48 & ,76 \\
\hline \multirow[t]{2}{*}{$\mathrm{ReO3}$} & ¿Cuán frecuentemente reza usted? & 2,98 & ,98 &, 51 & ,78 \\
\hline & Escala completa & 9,11 & 2.35 & & \\
\hline \multicolumn{2}{|r|}{$\begin{array}{l}\text { Fundamentalismo Cristiano } \\
\text { (Feldman \& Stenner, 1997) }\end{array}$} & $\mathbf{M}$ & SD & & \\
\hline RF01 & $\begin{array}{l}\text { La Biblia es la palabra de Dios y debe ser tomada } \\
\text { literalmente, palabra por palabra }\end{array}$ & 3,21 & ,96 & & \\
\hline
\end{tabular}


Estas tres escalas mostraron una adecuada consistencia. Para la escala de identidad nacional el alfa de Cronbach fue de 0,84 y los coeficientes de correlación fueron superiores a 0,65. Para la escala de nacionalismo el alfa de Cronbach fue de 0,81 y los coeficientes de correlación fueron superiores a 0,47. Para la escala de patriotismo el alfa de Cronbach fue de 0,70 y los coeficientes de correlación fueron superiores a 0,31 .

Por otra parte dos medidas de religión fueron utilizadas. Para medir la religiosidad se empleó la Medida Religiosa de la Juventud (Lindner y Moore, 2002). Religiosidad es medida por este índice como la práctica de ser religioso (ir a la iglesia, orar y adscribirse a los valores de las creencias religiosas). Esta medida está compuesta también por una escala de Likert con cuatro puntos de respuesta que van de 1 nada importante / nunca, hasta 4 muy importante / muchas veces. Esta escala mostró un alfa de Cronbach de 0,70 y coeficientes de correlación iguales o superiores a 0,48 . Adicionalmente se usó un ítem para medir fundamentalismo religioso cristiano tomado del trabajo de Feldman \& Stenner (1997).

Todas las escalas fueron sometidas a un análisis exploratorio de factores mostrando un único factor de solución. Los autovalores de las escalas fueron: identidad nacional 2,28 para un $76,1 \%$ de la varianza explicada; nacionalismo 3,25 para un $54,1 \%$ de la varianza explicada; patriotismo 2,4 para un $40 \%$ de la varianza explicada; y afiliación religiosa 1,8 para un $62,5 \%$ de la varianza explicada.

Además todos los instrumentos fueron sometidos al procedimiento de la "re-traducción" (John y Benet-Martínez, 2000) o back translation que consiste en la traducción de los ítem de un idioma a otro, para posteriormente volver a traducir el resultado al idioma original. Las diferencias en las traducciones fueron discutidas entre traductores. A pesar de ello la traducción de los ítems no se llevó a cabo con la intención de plasmar literalmente los contenidos de la versión original sino de mostrar equivalencias en el contexto costarricense.

Como variables demográficas se incluyó la identificación religiosa con las opciones católica, cristiana evangélica, judía, testigo de Jehová, mormón, sin religión o ateo y otra; nivel educativo, sexo y clase social.

El análisis estadístico de los datos fue realizado empleando el programa SPSS 11.5 y AMOS 5.0.

\section{Resultados}

Con el fin de evaluar la existencia de diferencias significativas en los resultados de las escalas según los datos sociodemográficos de los participantes se realizaron pruebas $\mathrm{t}$ (T-Test) y análisis de variancia de un factor (ANOVA One $W a y)$. 
Las pruebas t para las escalas por estatus socioeconómico, distrito de procedencia y sexo, no mostraron diferencias significativas para ninguna de las dimensiones estudiadas. Así mismo los análisis de varianza de un factor no mostraron diferencias para las escalas usando como variable independiente la variable educación. En cambio la prueba $\mathrm{t}$ para las diferencias entre padres e hijos mostró diferencias significativas para el índice de religiosidad $(\mathrm{t}=1,99, \mathrm{df}=162, p<0.05)$ denotando que los padres $(\mathrm{M}=$ 9.6; $\mathrm{SD}=2.23)$ puntúan significativamente más alto que los hijos $(\mathrm{M}=8.85$; $\mathrm{SD}=2.38$ ) en este índice. Las demás medidas no mostraron diferencias significativas para progenitores e hijos/as.

El análisis de variancia de un factor mostró que para el índice de religiosidad $(\mathrm{F}(3,158)=7.25 ; p<0.001)$, el ítem de fundamentalismo $(\mathrm{F}(3$, $158)=10.41 ; p<0.001)$, la escala de nacionalismo $(\mathrm{F}(3,158)=4.68 ; p<$ $0.01)$, y la escala de identidad nacional $(\mathrm{F}(3,158)=2.89 ; p<0.05)$ se presentaban diferencias significativas usando como variable independiente la auto identificación religiosa; mientras que para la escala de patriotismo no se encontraron diferencias estadísticamente significativas por identificación religiosa $(\mathrm{F}(3,158)=0.55 ; p=0.64)$.

Por esta razón se realizaron análisis post hoc usando para tal fin la prueba de Scheffe con el fin de evidenciar tales diferencias. En las dos medidas de religiosidad se encontraron diferencias significativas entre las personas que se identificaron como católicos y evangélicos con las personas que se identificaron como ateos (para fundamentalismo: diferencia de promedios católicos-ateos $=2.16, \mathrm{SD}=0.45 p<0.0001$; diferencia de promedios evangélicos-ateos $=2.51, \mathrm{SD}=0.46 p<0.0001 ;$ para el índice de religiosidad: diferencia de promedios católicos-ateos $=4.32, \mathrm{SD}=1.13 p<$ 0.01 ; diferencia de promedios evangélicos-ateos $=4.92, \mathrm{SD}=1.16 p<$ 0.001). Este resultado no es sorprendente debido a que las personas que se identificaron como ateos consecuentemente puntuaron muy bajo en las medidas de religiosidad.

De manera similar, en la escala de nacionalismo se encontró una diferencia significativa entre católicos y evangélicos con respecto a los participantes que se identificaron como ateos (diferencia de promedios católicos-ateos $=5.22, \mathrm{SD}=1.58 p<0.05$; diferencia de promedios evangélicos-ateos $=4.73, \mathrm{SD}=1.62 p<0.05)$. Este resultado muestra que los católicos y evangélicos que respondieron la encuesta muestran puntajes significativamente más altos en la escala de nacionalismo que los respondientes que se identificaron como ateos. Tal resultado representa un apoyo a la cuarta hipótesis de esta investigación, sin embargo, no se puede considerar una evidencia concluyente. Finalmente, en la escala de identidad nacional se encontraron diferencias entre los participantes que se identificaron como evangélicos y los que señalaron adscribirse a otra fe diferente a la católica, cristiana evangélica, judía, testigos de Jehová o 
mormón (diferencia de promedios evangélicos-otra religión $=2.04, \mathrm{SD}=$ $0.69, p<0.05)$.

Este resultado muestra que los católicos y evangélicos que respondieron la encuesta muestran puntajes significativamente más altos en la escala de nacionalismo que los respondentes que se identificaron como ateos. Finalmente, en la escala de identidad nacional se encontraron diferencias entre los participantes que se identificaron como Evangélicos y los que señalaron adscribirse a otra fe diferente a la Católica, Cristiana Evangélica, Judía, Testigos de Jehová o Mormón (diferencia de promedios Evangélicos-Otra religión $=2.04, \mathrm{SD}=0.69, p<0.05)$. Las diferencias significativas entre ateos y creyentes en las dimensiones de nacionalismo e identidad nacional representan un apoyo a la tercera hipótesis de esta investigación, sin embargo, no se puede considerar una evidencia concluyente.

Tabla 2

Correlaciones entre identidad nacional, nacionalismo, patriotismo, religiosidad y fundamentalismo

\begin{tabular}{lccccc}
\hline & A & B & C & D & E \\
\hline A. Identidad Nacional & 1 & $0,68^{* *}$ & $0,47^{* *}$ & $0,32^{* *}$ & $0,21^{* *}$ \\
B. Nacionalismo & & 1 & $0,57^{* *}$ & $0,29^{* *}$ & $0,17^{*}$ \\
C. Patriotismo & & & 1 & $0,15^{*}$ & 0,00 \\
D. Religiosidad & & & & 1 & $0,44^{* *}$ \\
E. Fundamentalismo & & & & & 1
\end{tabular}

Nota: ${ }^{*} p<0,05 \mathrm{y} * * p<0,01 . \mathrm{N}=164$.

Por otra parte, las correlaciones entre las dimensiones muestran que la escala de nacionalismo presenta correlaciones significativas con todas las variables estudiadas, principalmente con identidad nacional, patriotismo, y religiosidad. En menor medida aunque también estadísticamente significativa se correlaciona con fundamentalismo. Todas estas correlaciones son positivas.

Como se puede observar la identidad nacional muestra correlaciones positivas con nacionalismo y patriotismo, lo cual implica que las personas que reportan una mayor identidad nacional muestran también más patriotismo y nacionalismo; aunque es de destacar que la correlación más fuerte se presenta entre identidad nacional y nacionalismo. Por otro lado, 
como era de esperarse ambas medidas de religiosidad se relacionan fuertemente entre sí.

La escala de identidad nacional se encuentra también relacionada positivamente con el índice de religiosidad y la medida de fundamentalismo. Las personas cuyos puntajes son más altos en identidad nacional tienen también puntajes más altos en afiliación religiosa y fundamentalismo.

Patriotismo es la actitud que muestra correlaciones más pobres con las variables religiosas. Con afiliación religiosa la correlación es significativa pero baja y con fundamentalismo no hay ninguna relación.

Con estos resultados se confirma la primera parte de la hipótesis uno debido a que el índice de religiosidad se correlaciona positiva y altamente significativamente con las medidas de nacionalismo e identidad nacional en la muestra estudiada. Con respecto a las correlaciones con fundamentalismo, si bien esta medida correlaciona de manera positiva y altamente significativa con identidad nacional, con nacionalismo la correlación es, si bien significativa y positiva, baja. Por lo que se puede hablar en este caso solamente de una tendencia. Adicionalmente, el resultado de la correlación entre fundamentalismo y patriotismo fue de cero lo que era de esperar desde un punto de vista teórico pues ambas variables implican posiciones cognoscitivas diametralmente opuestas.

Para probar la tercera hipótesis se procedió en un primer momento a probar dos modelos de regresión lineal usando nacionalismo como variable dependiente. En el primero de ellos la medida de fundamentalismo y el índice de religiosidad fueron empleados como variables predictoras con el fin de evaluar el peso de estas dos variables religiosas sobre el nacionalismo; mientras que en el segundo modelo se agregaron a la lista de variables predictoras la identidad nacional y patriotismo con el fin de comparar el poder predictivo de las variables relacionadas con actitudes políticas y las variables religiosas.

En el modelo 1 se realizó una regresión lineal simple simultanea del índice de religiosidad y la medida de fundamentalismo sobre nacionalismo que mostró un una relación lineal significativa entre las variables, aunque el coeficiente de determinación indica que el monto de la variancia explicado es bajo $\left(F(2,161)=7.92, p<0.001, R^{2}=0.09\right)$. El índice de religiosidad se constituyó en un predictor significativo en el modelo $(\beta=0.27, p<0.001)$ a diferencia de fundamentalismo cristiano $(\beta=0.05, p=0.54)$. Seguidamente, se procedió a evaluar el efecto de cada una de las dos medidas de religiosidad sobre la medida de nacionalismo. La regresión de fundamentalismo sobre nacionalismo muestra que ambas variables se relacionan linealmente ( $F$ (1, $162)=4.88, p<0.05)$ pero con un efecto moderado sobre la variable dependiente $(\beta=0.17, p=0.03)$. Por su parte el índice de religiosidad 
mostró una relación lineal más fuerte con el nacionalismo $(\mathrm{F}(1,162)=15.54$, $p<0.001)$ y un efecto de una mayor magnitud $(\beta=0.30, p<0.001)$.

De tal manera, aunque se comprueba la condición de predictor del índice religiosidad sobre nacionalismo, se debe señalar que fundamentalismo no constituye un adecuado predictor de la variable dependiente y que el modelo en general explica poco el comportamiento de la variable dependiente.

En el segundo modelo, donde se usaron como variables independientes el índice de religiosidad, fundamentalismo, la escala de identidad nacional y la de patriotismo, el coeficiente de determinación mostró que nacionalismo como variable dependiente es explicada en un $62 \%$ por las variables independientes en su conjunto $\left(R^{2}=0,62\right)$. Los análisis demostraron que el modelo muestra una relación lineal significativa $\mathrm{F}(4,159)=57.261, p<$ 0,001. Sólo tres de las variables independientes contribuyen significativamente a predecir nacionalismo. En orden de importancia: identidad nacional $(\beta=0.51, p<0.0001)$, patriotismo $(\beta=0.35, p<0.0001)$ y el índice de religiosidad $(\beta=0.13, p<0.0001)$. La medida de fundamentalismo no predice la variable dependiente de una manera significativa $(\beta=0.008, p=0.89)$. De esta manera se puede afirmar que en esta muestra los valores en la escala de nacionalismo son afectados por variables como identidad nacional, patriotismo y afiliación religiosa, pero no así por la medida de fundamentalismo. Por lo tanto la hipótesis 3 se confirma de una manera parcial.

Con el fin de evaluar la influencia del fundamentalismo religioso y la identidad nacional, se procedió a evaluar en un tercer modelo de regresión lineal con el fin de conocer cuáles variables tienen un efecto sobre la identidad nacional. Para ello la escala de identidad nacional se tomó como dependiente $y$ el resto de las escalas y medidas como variables independientes. El modelo 3 mostró un adecuado ajuste $(F(4,159)=39.39$, p $\left.<0.0001 ; R^{2}=0.48\right)$ aunque en términos comparativos solamente nacionalismo $(\beta=0.57, \phi<0.0001)$ mostró ser una variable significativamente predictora. Las demás variables mostraron un efecto bajo (patriotismo $\beta=0.12, p=0.07$; índice de religiosidad $\beta=0.10, p=0.1$ ) o prácticamente nulo (fundamentalismo $\beta=0.06, p=0.29$ ). Este resultado llama la atención pues indica que en la muestra estudiada la identidad nacional muestra, desde el punto de vista de las variables políticas, un componente principalmente nacionalista y no patriótico. Por otro lado se observa que de nuevo la medida de fundamentalismo tiene un efecto insignificante sobre la identidad nacional aunque el índice de religiosidad sí tiene un efecto relativamente pobre, en comparación con las variables de actitudes políticas, sobre la identidad nacional. Sin embargo atendiendo a los 
resultados de la prueba de diferencias de promedios para la escala de identidad nacional entre católicos y ateos, así como la influencia de la religiosidad sobre nacionalismo, podemos sospechar de algún efecto indirecto de ésta variable sobre las identificaciones nacionales. Por ello se propone como hipótesis que la religiosidad podría tener un efecto mediado por nacionalismo sobre la identidad nacional. Con el objetivo de probar esta relación entre religiosidad, nacionalismo e identidad nacional se elaboró un modelo de mediación. Para ello se tomó a religiosidad, medido por el índice de religiosidad como variable exógena, nacionalismo como variable mediadora $^{3}$, e identidad nacional como variable criterio. La evaluación del modelo mediacional se llevó a cabo por medio de AMOS 5.0 siguiendo el procedimiento para evaluar mediación por medio de modelos de ecuaciones estructurales expuesto por Holmbeck (1997).

Para este fin se construyó en primera instancia un modelo de medición en la que evaluaron las correlaciones entre las tres variables latentes (Identidad Nacional, Nacionalismo y Religiosidad). Para estimar el modelo se usó el método de máxima similitud (maximun likehood). La normalidad univariada y multivariada fue evaluada por medio de AMOS 5.0, y siguiendo los criterios de Finch, West y MacKinnon (1997). No se identificaron problemas de valores extremos (outliers) a nivel univariado o multivariado. El modelo fue identificado y presentó un adecuado ajuste ${ }^{4}\left(X^{2}=91.67\right.$, DF $\left.=51, \mathrm{P}=0.00, \mathrm{X}^{2} / \mathrm{DF}=1.79, \mathrm{CFI}=0.94, \mathrm{RMSEA}=0.07 ; \mathrm{SRMR}=0.05\right)$. Todos los factores latentes se mostraron una adecuada carga factorial (valores en un rango de 0.45 y 0.84 ) sobre las variables observadas. Las correlaciones entre los factores latentes fueron asimismo significativas (correlaciones entre $\mathrm{r}=0.41 \mathrm{y} \mathrm{r}=0.88$ ).

Con estos resultados se procedió a elaborar 2 modelos de ecuaciones estructurales para probar la influencia de la religiosidad sobre la identidad nacional usando como mediador al nacionalismo. En el primer modelo, identificado aquí como modelo no mediacional (ver figura 1), se establecieron regresiones de religiosidad y nacionalismo sobre identidad nacional y de religiosidad sobre nacionalismo. Los resultados mostraron un adecuado ajuste $\left(\mathrm{X}^{2}=91.67, \mathrm{DF}=51, \mathrm{P}=0.000, \mathrm{X}^{2} / \mathrm{DF}=1.79, \mathrm{CFI}=\right.$ 0.94$, RMSEA $=0.07$, Pclose $=0.07 ;$ SRMR $=0.05)$, sin embargo, la influencia directa de religiosidad sobre identidad nacional fue no significativa,

\footnotetext{
${ }^{3}$ Teóricamente una relación de mediación implica que un variable independiente no tienen efecto sobre la variable dependiente a menos que en medio de tal relación se incluya una variable mediadora la cual ve intensificado su efecto sobre la variable dependiente.

4 Para evaluar el ajuste general del modelo se siguen los criterios de Hu \& Bentler (1999) donde se espera un Chi cuadrado no significativo (aunque este criterios es muy influenciable por el tamaño de la muestra y la complejidad del modelo), un $\mathrm{X}^{2} / \mathrm{DF} \leq 3$, un CFI $\geq 0.95$, un RMSEA ("Root mean square of aproximation") $\leq 0.06-0.08$, y un SRMR ("Standarized root mean residual") $\leq 0.08$
} 
( $\beta=0.05$, n.s.) mientras que el nacionalismo predecía muy fuertemente la identidad nacional $(\beta=0.87, p<0.001)$. La regresión de religiosidad sobre nacionalismo fue también significativa $(\beta=0.41, p<0.001)$.

En el segundo modelo, llamado modelo mediacional, se colocó a nacionalismo como variable mediadora entre religiosidad e identidad nacional. Este segundo modelo mostró una mejoría en el ajuste global del modelo $\left(\mathrm{X}^{2}=92.07, \mathrm{DF}=52, \mathrm{P}=0.001, \mathrm{X}^{2} / \mathrm{DF}=1.77, \mathrm{CFI}=0.95\right.$, RMSEA $=0.06$, Pclose $=0.09 ;$ SRMR $=0.05)$ pero con la diferencia de que por el efecto de la religiosidad sobre el nacionalismo, esta variable incremento su efecto sobre identidad nacional $(\beta=0.90, p<0.001$ ) (ver Figura 1).

Para corroborar la magnitud del efecto de religiosidad sobre identidad nacional se calcularon los efectos indirectos y directos (Fox, 1980; Maruyama, 1998) entre las variables. Como se puede observar en la Tabla 3, el efecto estandarizado de la medida de religiosidad sobre la medida de identidad nacional a través de la medida de nacionalismo es de $\beta=0.38$, lo cual implica un efecto mediado muy fuerte.

Tabla 3

Efectos directos e indirectos entre los factores latentes en el modelo mediacional

\begin{tabular}{|lcccc|}
\hline & \multicolumn{2}{c}{ Religiosidad } & \multicolumn{2}{c|}{ Nacionalismo } \\
& $\mathrm{D}$ & $\mathrm{I}$ & $\mathrm{D}$ & $\mathrm{I}$ \\
\hline Identidad Nacional & $\beta=.00$ & $\beta=.38$ & $\beta=.90$ & $\beta=.00$ \\
Nacionalismo & $\beta=.42$ & $\beta=.00$ & $\beta=.00$ & $\beta=.00$ \\
\hline $\begin{array}{l}\text { Todas las regresiones presentadas en valores estandarizados. } \mathrm{D}=\text { Efecto directo, I = Efecto } \\
\text { indirecto. }\end{array}$ \\
\hline
\end{tabular}


La religión como predictor de las actitudes hacia la nación

Figura 1. Resultados del modelo mediacional y el modelo no mediacional

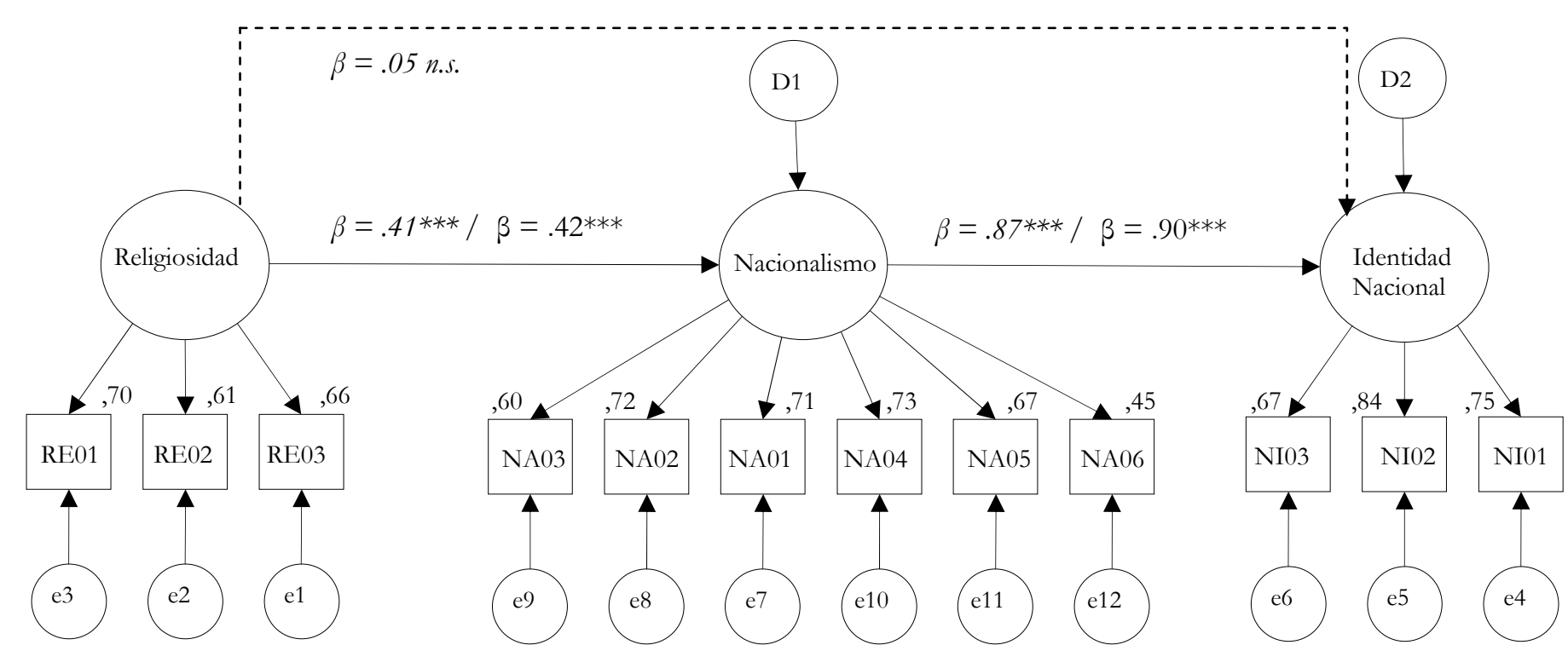

*** $=$ Nivel de significación $0.001, \mathrm{n} . \mathrm{s}=$ No significativo. En itálica pesos de regresión del modelo no mediacional. Sin itálica, modelo mediacional. Línea punteada: regresión adicional en el modelo no mediacional. Todos los pesos de regresión entre los factores latentes y las variables observables se presentan en valores estandarizados y son significativos al nivel 0.001 . Todas las varianzas de las variables latentes difieren de 0 a un nivel de significancia del 0.001 . 
Como manera alternativa para probar si el efecto indirecto de la variable índice de religiosidad sobre la variable dependiente identidad nacional, vía la variable medidora nacionalismo es significativamente diferente de 0, se aplicó el test Sobel (Baron \& Kenny, 1986). El resultado mostró que el efecto indirecto de la religiosidad sobre identidad nacional vía nacionalismo como mediador era significativamente diferente de 0 (Sobel test: $3.40, p<0.001$ ), con lo que se comprueba la fortaleza del efecto indirecto de religiosidad sobre identidad nacional.

Finalmente con el objetivo de evaluar si la muestra tenía una cantidad adecuada de participantes para sostener este modelo, se uso la medida de Hoelter ${ }^{5}$ (Byrne, 2001). Tal medida indicó que el modelo solamente era válido con un nivel de significación de $p<0.01$ si la muestra estaba integrada al menos por 140 personas. Dado que la muestra cuenta con 164 personas el modelo puede ser aceptado con un nivel de significación de $p<0.01$.

De tal manera podemos aceptar que la religiosidad tiene un efecto sobre identidad nacional cuando este efecto es mediado por nacionalismo, aunque de forma directa no existe tal efecto entre religiosidad e identidad nacional. De tal manera la hipótesis cuatro puede aceptarse de una manera parcial.

\section{Discusión}

Como lo demuestran los resultados se presentó una correlación significativa entre variables como nacionalismo, identidad nacional, religiosidad y fundamentalismo religioso. Esta relación no fue así para la variable patriotismo con religiosidad y fundamentalismo religioso, donde patriotismo se correlacionó con afiliación religiosa pero en menor medida y no mostró una correlación significativa con fundamentalismo con lo que se confirman la primera y segunda hipótesis. La tercera hipótesis solamente se ve confirmada parcialmente pues aunque la religiosidad es un predictor de nacionalismo, el fundamentalismo religioso no se comporta de esta misma manera. La cuarta hipótesis se corroboró también de una manera parcial pues fundamentalismo no muestra efectos importantes sobre identidad nacional. Religiosidad, sin embargo, mostró tener efectos sobre identidad nacional cuando tal relación fue mediada por nacionalismo.

El fundamentalismo no se presenta como predictor de nacionalismo en el modelo. Estos resultados están en contradicción con lo esperado desde un punto de vista teórico. Teóricamente es esperable que fundamentalismo y

\footnotetext{
${ }^{5}$ La "N" crítica de Hoelter indica una relación entre el ajuste del modelo y el tamaño de la muestra del estudio. Se asume que los resultados de ajuste de un modelo son correctos si la muestra es mayor que la " $\mathrm{N}$ " crítica. El programa AMOS 5.0 calcula la "N" con niveles de significancia de .05 y .01. Más información en Hoelter (1983).
} 
nacionalismo variables además de estar correlacionadas, muestren al fundamentalismo como predictor de nacionalismo. Lo anterior sigue la lógica de que a más intensidad de creencia religiosa mayor nacionalismo. Por ello, siendo que el fundamentalismo religioso es una posición extrema en la que se vincula el individuo con su creencia religiosa seria esperable que esta variable se comportara también como un predictor de nacionalismo.

Una posible explicación de por qué el fundamentalismo no se comporta como predictor significativo de nacionalismo sería que el fundamentalismo religioso implica una forma de relación con la creencia religiosa que se encuentra "por encima" de otras vinculaciones posibles, entre ellas el discurso nacionalista. La participación del fundamentalista en el discurso nacionalista sería secundaria en la medida que para este grupo su vinculación grupal es diferente al contexto político. Sin embargo, esta investigación no puede dar cuenta de la anterior especulación, la cual debería ser objeto de análisis por otros medios.

Lo que sí demuestran los resultados con esta muestra es que efectivamente la religiosidad es un factor que incide en el nacionalismo. Esta relación apoyaría la tesis de que el nacionalismo en Costa Rica ha sido construido conectando discursos religiosos y discursos nacionalistas. Lo anterior implica que en el nivel psicosocial se puede encontrar alguna evidencia a los planteamientos antropológicos e historiográficos, tanto en lo que respecta a las teorías generales sobre el nacionalismo como en las investigaciones costarricenses sobre este aspecto.

Por otra parte, un resultado también importante es que la identidad nacional costarricense parece responder más a una posición nacionalista que a una posición patriótica. Esto implica que los costarricenses se vinculan a su nación de una manera preponderantemente ideológica y acrítica reproduciendo discursos nacionalistas que entre otras implicaciones pueden devenir en el rechazo a los otros y la autopercepción de superioridad. La relación de los costarricenses con su costarriqueñidad no parece atravesar la preocupación real por las instituciones de la sociedad o la manifestación de posiciones críticas para con el Estado, sino más bien asume un discurso nacionalista. Esta posición se ve incrementada por la participación de la religión, la cual, a un nivel psicosocial conforma un frente común con el nacionalismo.

Tales resultados deberían tener implicaciones en la investigación social en el futuro. La primera de ellas y tal vez la más obvia es tematizar más ampliamente las relaciones entre nacionalidad y religión. Avances en este sentido ya han sido presentados en el trabajo de González (1994) antes referido y en algunos resultados de la investigación historiográfica. Sin embargo, los resultados aquí expuestos deben ser valorados solamente como un primer paso en el esclarecimiento de esta relación. Tal inquietud implica trabajar con otros tipos de muestras y otros tipos de instrumentos. 
Un trabajo muy pertinente es la comparación de diversos grupos en estos aspectos, como son grupos religiosos versus grupos no religiosos y comparaciones entre diferentes grupos religiosos entre sí. Esto permitiría esclarecer por ejemplo si las diferentes adscripciones religiosas tienen un peso específico en el nacionalismo o no, ya que según la tesis de González, la nación costarricense se ha construido a partir de una relación con la iglesia católica costarricense. Por lo tanto, grupos religiosos diferentes al catolicismo y que por consiguiente están fuera de la oficialidad, podrían presentar a su vez posiciones nacionalistas que se lograrían distanciar o ser divergentes de las vinculaciones a la nación. Los resultados de comparaciones entre medias parecen apoyar este aspecto, sin embargo, tales resultados no se podrían considerar definitivos.

Otro aspecto necesario es estudiar la manera en la que diferentes dimensiones de la religiosidad se vinculan al nacionalismo. En este sentido resultaría necesario el empleo de diferentes instrumentos para medir la religiosidad. La medida de religiosidad empleada en esta investigación si bien está centrada en la evaluación de importantes dimensiones del ser religioso (rezar, importancia de la religión, asistir a actividades religiosas y fundamentalismo religioso) deja de lado la posibilidad de análisis detallados en los que se relacionen las características de vinculación religiosa con las actitudes nacionalistas. En este sentido, los intentos por diferenciar las actitudes religiosas como es el caso de las escalas de religiosidad intrínseca, religiosidad extrínseca (Allport, 1966), la escala de búsqueda (Batson, Schoenrade, \& Ventis, 1993) o la Escala de Creencias Poscríticas (Fontaine, Duriez, Luyten, \& Hutsebaut, 2003) constituyen una importante alternativa para desmontar cuales posiciones religiosas son más propensas a estimular el nacionalismo que otras. De esta manera una evaluación general de la religiosidad si bien implica una buena aproximación a la relación religión nacionalismo carece de la posibilidad de distinguir al interior de la religiosidad misma el aporte específico de las diversas posiciones a esta relación.

Por otra parte los resultados expuestos estarían parcialmente en conflicto con las opiniones de Armstrong (1997) quien plantea que las relaciones entre nacionalismo y religión se hacen evidentes principalmente en los procesos de grandes transformaciones sociales cuando se presentan amenazas a la identidad. El hallazgo de conexiones entre religiosidad y nacionalismo sin contar con un marco social de una amplia transformación social permite hipotetizar que tal conexión debería ser manifiesta en otros aspectos de la cotidianidad y por lo tanto podría ser observable en la vida cotidiana. Algunas posibilidades para la constatación de esta tesis es la investigación en escenarios cotidianos en los que se pone de manifiesto diferentes aspectos de las identidades sociales, y en especial aquellas que están vinculadas con la nacionalidad. 
Sin embargo, como ya se señaló, en este articulo el análisis se ha enfocado solamente en uno de los componentes del nacionalismo, y quizás en uno de los menos evidentes demostrando relaciones positivas. Esto lo que hace patente es la necesidad de desarrollar más investigaciones que permitan identificar otros componentes nacionalistas en la Costa Rica actual. En este sentido los diferentes factores que potencian el nacionalismo en Costa Rica deben ser descompuestos y analizados, tarea que sin duda desborda las posibilidades de la psicología social y la psicología política, y requiere de diversos abordajes metodológicos y disciplinarios.

\section{Referencias}

Adorno, T. (1950). The authoritarian personality. New York : Harper \& Row.

Allport, G. (1954). The nature of prejudice. Cambridge: Addison-Wesley.

Allport, G. (1966). Religious context of prejudice. Journal of Scientific Study of Religion, 5, 447457.

Altemeyer, B. (2003). Why religious fundamentalist tend to be prejudiced? The International Journal for the Psychology of Religion. 13, 17-28.

Anderson, B. (1991). Imagined communities. New York: Verso.

Armstrong, J. (1997). Religious nationalism and collective violence. Nations and Nationalism. 3, 597-606.

Baron, R. \& Kenny, D. (1986). The moderator-mediator variable distinction in social psychology research: Conceptual, strategic and statistical considerations. Journal of Personality and Social Psychology, 51, 1173-1182.

Batson, D., Schoenrade, P., \& Ventis, L. (1993). Religion and the individual. A social psychological perspective. Oxford: Oxford University Press.

Baumann, G. (2001). El enigma multicultural. Un replanteamiento de las identidades nacionales, étnicas y religiosas. Barcelona: Paidós.

Blank, T. \& Shmidt, P. (2003). National identity in a united Germany: Nationalism or patriotism? An empirical test with representative data. Political Psychology. 24, 289-312.

Burris, C.; Branscome, N; Jackson, L. (2000). For God and country. Religion and endorsement national stereotypes. Journal of Cross-Cultural Psychology, 31, 517-527.

Byrne, B. (2001). Structural equation modeling with AMOS. Basic concepts, applications, and programming. New Jersey: Erlbaum.

Cohrs, C. (2005). Patriotismus. Sozialpsychogische Aspekte. Zeitschrift für Sozialpsychologie, 36, 311.

De Figueredo, R. \& Elkins, Z. (2003). Are patriots bigots? An inquiry into the vices of ingroup pride. American Journal of Political Science, 47, 171-188.

Duriez, B., Luyten, P., Snauwaert, B., \& Hutsebaut, D. (2002). The importance of religiosity and values in predicting political attitudes: Evidence for the continuing importance of religiosity in Flanders. Mental Health, Religion and Culture, 5, 35-59.

Eisinga, R., Felling A., \& Peters, J. (1990a). Religious belief, church involvement, and ethnocentrism in the Netherlands. Journal of Scientific Study of Religion, 29, 54-75.

Eisinga, R., Felling, A. \& Peters, J. (1990b). Church involvement, prejudice and nationalism: A research note on the curvilinear relationship between church involvement and ethnocentrism in the Netherlands. Review of Religious Research, 31, 417-433.

Feldman, S. \& Stenner, K. (1997). Perceived threat and authoritarianism. Political Psychology, 18, 741-770. 


\section{José Miguel Rodríguez-García}

Feschbach, S. (1994). Nationalism, patriotism, and aggression. A clarification of functional differences. En J. Huesmann (Ed). Aggressive behavior. Current perspectives. New York: Plenum Press.

Finch, J., West, S., \& MacKinnon, D. (1997). Effects of the sample size and nonnormality on the estimation of mediated effects in latent variable models. Structural Equation Modelling, 4, 87-107.

Fontaine, J., Duriez, B., Luyten, P. \& Hutsebaut, D. (2003). The internal structure of the PostCritical Belief scale. Personality and Individual Differences, 35, 501-518.

Fox, J. (1980) Effect analysis in structural equation models. Sociological Methods and Research, 9, 3-28.

Friedland, R. (2001). Religious nationalism and the problem of collective representation. Annual Review of Sociology, 27, 125-152.

Gellner, E. (1989). Cultura, identidad y política. El nacionalismo y los nuevos cambios sociales. Barcelona: Gedisa.

Gellner, E. (1997). Naciones y nacionalismo. Madrid: Alianza.

Gellner, E. (1998). Nacionalismo. Barcelona: Destino.

González, A. (1994). Costa Rica, el discurso de la patria. San José, Costa Rica: Editorial de la Universidad de Costa Rica

Hjerm, M. (1998). National identities, national pride and xenophobia: A comparison of four western countries. Acta Sociologica, 41, 335-347.

Hobsbawm, E. (1991). Naciones y nacionalismo desde 1780. Barcelona: Crítica.

Hoelter, J. (1983). The analysis of covariance structures: Goodness-of-fit indices. Sociological Methods and Research, 11, 325-344.

Holmbeck, G. (1997). Toward terminological, conceptual, and statistical clarity in the study of mediators and moderators: Examples from the child-clinical and pediatric psychology literatures. Journal of Consulting and Clinical Psychology, 65, 599-610.

Hu, L. \& Bentler, P. (1999). Cutoff criteria for fit indexes in covariance structure analysis: Conventional criteria versus new alternatives. Structural Equation Modelling, 6, 1-55.

Hunsberger, B. \& Jackson, L. (2005). Religion, meaning, and prejudice. Journal of Social Issues, 61, 807-826.

John, O. \& Benet-Martínez, V. (2000). Measurement: Reliability, constructs validation, and scale construction. En H. Reis, \& C. Judd (Eds). Handbook of Research Methods in Social and Personality Psychology. U.S.A: Cambridge University Press.

Johnstone, R. (1991). Religion in society. A sociology of religion. New Jersey: Prentice Hall.

Kelman, H. (1997). Nationalism, patriotism, and national identity: Social-psychological dimensions. En D. Bar-Tal \& E. Staub, (Eds). Patriotism in the live of the individual and the nations. Chicago: Nelson-Hall.

Levinson, D. (1957). Authoritarian personality and foreign policy. Journal of Conflict Resolution, 1, 37-47.

Lindner, M. \& Moore, K. (2002). Predictors of religiosity among youth aged 17-22: A longitudinal study of national survey of children. Journal of Scientific Study of Religion, 41, 613-622.

Martin, J. \& Westie, F. (1959). The tolerant personality. American Sociological Review, 24, 521528.

Maruyama, G. (1998) Basics of structural equation modeling. California: Sage.

Mummendey, A. Klink, A., \& Brown, R. (2001). Nationalism and patriotism: National identification and outgroup rejection. British Journal of Social Psychology, 40, 159-172.

Rockeach, M. (1960). The open and closed mind. Investigations into the nature of belief systems and personality systems. New York: Basic Books. 
Schatz, R. \& Staub, E. (1997). Manifestations of blind and constructive patriotism. Personality correlates and individual-group relations. En D. Bar-Tal \& E. Staub (Eds). Patriotism in the live of the individual and the nations. Chicago: Nelson-Hall.

Schatz, R., Staub, E. \& Lavine, H. (1999). On the varieties of national attachment: blind versus constructive patriotism. Political Psychology, 20, 151-174.

Wegener, D. \& Fabrigar, L. (2000). Analysis and design for nonexperimental data. Addressing causal and noncausal hypotheses. En H. Reis \& C. Judd, (Eds). Handbook of Research Methods in Social and Personality Psychology. U.S.A: Cambridge University Press.

Recibido: 18 de mayo de 2005 Aceptado: 12 de diciembre de 200 
